\title{
The incidence of nausea in the absence of vomiting in patients receiving intravenous chemotherapy
}

\author{
Teresa Smit^, Irma Kotze^, Jesslee du Plessis^ \\ Medicine Usage in South Africa (MUSA), School of Pharmacy, Faculty of Health Sciences, North-West University, Potchefstroom, South Africa \\ Contributions: (I) Conception and design: All authors; (II) Administrative support: All authors; (III) Provision of study materials or patients: All \\ authors; (IV) Collection and assembly of data: All authors; (V) Data analysis and interpretation: All authors; (VI) Manuscript writing: All authors; (VII) \\ Final approval of manuscript: All authors. \\ Correspondence to: Jesslee du Plessis, MD. Private Bag X6001, Potchefstroom, 2520, South Africa. Email: jesslee.duplessis@nwu.ac.za.
}

\begin{abstract}
Background: The magnitude of the incidence and impact of nausea on patients receiving intravenous chemotherapy seems to be underestimated by healthcare professionals. Development of effective anti-emetic treatment has contributed to the resolution of chemotherapy-induced nausea and vomiting (CINV). However, there is a concern that vomiting has been the initial focus of anti-emetic research and nausea was perceived as a secondary endpoint. Through focusing on the incidence of nausea independently of the incidence of vomiting, valuable information has been gained on this distressing side effect, including identifying patient risk factors contributing to the increased experience of nausea.

Methods: The study followed a prospective, observational study design in a private oncology centre in Johannesburg, South-Africa. Ethical approval was obtained before commencement of the study, followed by the recruitment of one hundred patients over a seven-month period. Patient-reported outcome measures (PROMs) were used to measure nausea with an amended version of the Multinational Association of Supportive Care in Cancer antiemesis tool (MAT). Patients documented information in their diaries on the incidence, duration and severity of nausea, during the acute phase (0-24 hours), the delayed phase (25-120 hours), day 7 and day 10 after infusion of chemotherapy, with episodes of vomiting being recorded as a secondary endpoint. The demographic and clinical variables of the subjects, as well as patient risk factors known to cause CINV, were tabulated and summarised using descriptive statistics.
\end{abstract}

Results: The population consisted of 68 females and 27 males with a mean age of 57 years (25-84 years). The emetogenicity of chemotherapy regimens administered were well represented with $26.3 \%$ low emetogenic chemotherapy, $25.3 \%$ moderately emetogenic chemotherapy and $48.4 \%$ highly emetogenic chemotherapy (HEC). Despite all patients receiving guideline consistent CINV prophylaxis, nausea was still experienced by $57.9 \%$ patients during cycle one, $50.6 \%$ patients during cycle two and $45.6 \%$ patients during cycle three. The incidence of patients experiencing nausea (in the absence of vomiting) was $35 \%$, compared to $2 \%$ of patients experiencing vomiting (in the absence of nausea). Patient characteristics with a known risk to impact CINV were documented, and significant impact in this study was found in female gender, age $<60$ years, history of motion sickness and history of morning sickness.

Conclusions: Guideline consistent CINV prophylaxes seem to have vomiting under control for most patients receiving intravenous chemotherapy. Nausea, however, still seems to be a persistent adverse event during treatment. Female gender, age $<60$ years, history of motion sickness and history of morning sickness increases the risk of experiencing nausea. A different approach is needed to manage nausea in the clinic setting, along with standardised tools to measure nausea specifically. More studies need to be done with nausea as the primary endpoint to address this ongoing medical need.

^ ORCID: Teresa Smit: http://orcid.org/0000-0001-5750-8815; Irma Kotze: http://orcid.org/0000-0002-0700-1300; Jesslee du Plessis: http://orcid.org/0000-0001-9189-393X. 
Keywords: Chemotherapy-induced nausea and vomiting (CINV); intravenous chemotherapy; persistent nausea

Submitted Nov 07, 2020. Accepted for publication Dec 08, 2020.

doi: $10.21037 /$ apm-19-453

View this article at: http://dx.doi.org/10.21037/apm-19-453

\section{Introduction}

Nausea and vomiting-caused by cytotoxic chemotherapy for cancer-remains one of the most feared adverse events of cancer treatment. This is known as chemotherapy-induced nausea and vomiting (CINV) (1). Chemotherapy-induced nausea is now recognised as a specific clinical problem which is often not optimally treated (2). It remains the most important unmet medical need regarding CINV (3). For many years, CINV has been regarded as a single entity (4). As one of the most serious treatment side effects in patients with cancer, CINV can significantly compromise patients' quality of life, but due to evidence-based research and guideline consistent CINV prophylaxis (GCCP), chemotherapyinduced vomiting can be prevented in the majority of patients $(5,6)$. Despite this, patients still experience nausea and its burden is often underestimated by the healthcare professionals $(5,7,8)$.

CINV presents in three phases. The acute phase occurs within 0-24 hours post-start of chemotherapy infusion, whereas the delayed phase occurs within 25-120 hours post-start of chemotherapy infusion (9). Delayed nausea is more difficult to manage than nausea in the acute phase, which occurs only after the patient has left the clinic (10). Anticipatory CINV is triggered in patients by taste, odour, sight and thoughts of anxiety due to a history of inadequate antiemetic prophylaxis in previous cycles, and it occurs before subsequent chemotherapy cycles (6).

Chemotherapy agents are classified into four different levels of emetogenicity: highly emetogenic chemotherapy (HEC) with $>90 \%$ risk of inducing CINV, medium emetogenic chemotherapy (MEC) with $>30-90 \%$ risk, low emetogenic potential (LEC) with $10-30 \%$ risk and minimal emetogenic potential ( $<10 \%$ risk) (11). Guidelines for prevention and treatment of CINV are based on this classification and consist of combinations of dexamethasone, 5-hydroxytryptamine $\left(5-\mathrm{HT}_{3}\right)$ receptor antagonists and neurokinin-1 (NK-1) receptor antagonists (12). Several studies have shown effective CINV prophylactic activity with olanzapine, an antipsychotic agent $(13,14)$. Chemotherapy-induced nausea is not life-threatening but has a vast impact on the patient and their treatment (15). Nausea leads to anorexia, malnutrition, dehydration and anxiety towards chemotherapy (14). This collectively plays a role in the overall recovery period of the patient and adds to the economic burden of medical care (16). The sensory experience of nausea and the associated physiological changes involve bi-directional interactions between the central nervous system, the autonomic nervous system and the endocrine system (2). The complex event of nausea can be simplified to a three-step process:

* Input signals from variety of emetic stimuli are sent from different parts of the body to the brain;

* The central pattern generator or vomiting centre receives and processes all these signals;

* Output signals are returned from the central pattern generator to different parts of the body (17).

The main areas involved in the receiving and processing of nausea and vomiting stimuli are the chemo trigger zone in the area postrema, the nucleus tractus solitarius and the central pattern generator in the reticular formation $(18,19)$. From here, output signals return to different parts of the body to create the somatic and autonomic symptoms accompanying nausea: pallor, sweating, salivation, swallowing, gagging, smooth muscle contraction, cramps and tachycardia (20).

Certain patient characteristics are documented as having an impact on the experience of nausea and vomiting. The female gender, patients younger than 60 years, patients with a history of motion sickness and patients with a history of morning sickness have a higher risk of experiencing CINV. Patients having a history of excessive alcohol intake ( $>4$ glasses per day) during the past two years are less likely to experience CINV (21). The emetogenicity of the chemotherapy contributes to the experience of nausea as an external factor $(3,22)$. Despite decades of research, nausea is still not clearly understood, and very little published literature is available on chemotherapy-induced nausea.

The research objectives discussed here includes:

* Comparing the incidence of nausea with the incidence of vomiting for all subjects;

* Documenting the possible patient-related 
Use the scale in the diary to indicate your nausea level out of ten with;

$$
\begin{gathered}
' 0 ’=\text { no nausea al all } \\
' 10 ’=\text { nausea at its worst }
\end{gathered}
$$

To illustrate the scale, look at the example below:

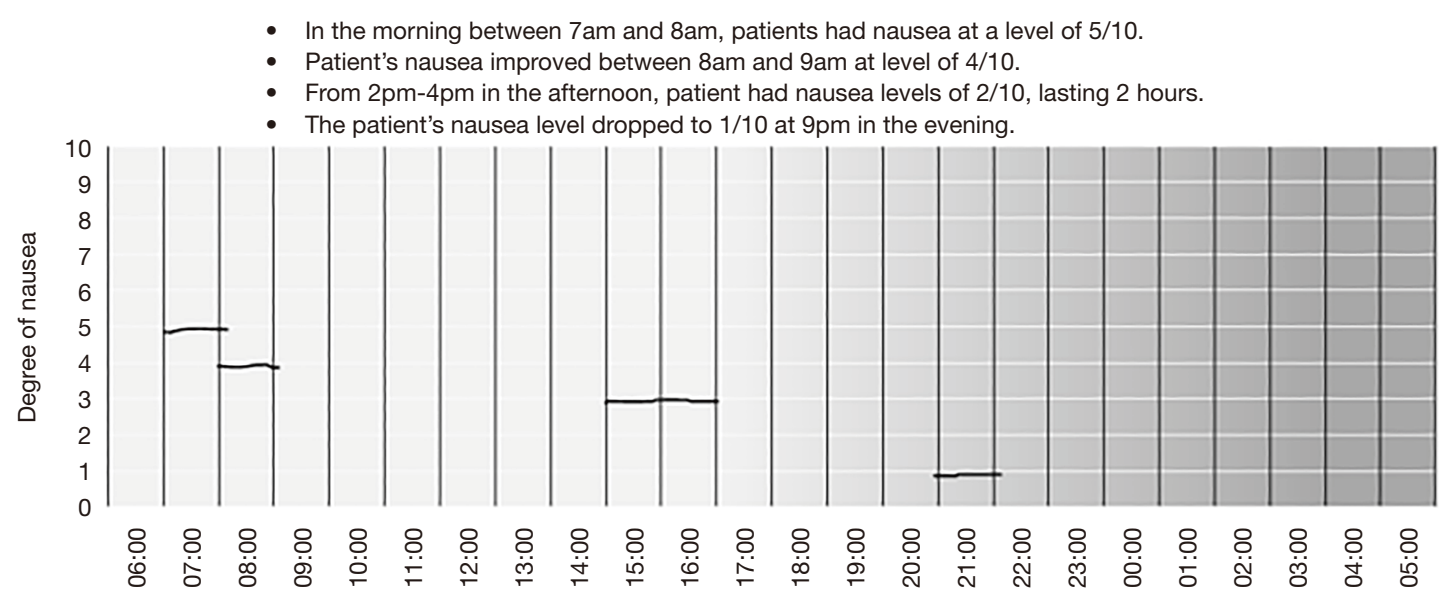

Figure 1 An extraction of the expanded version of MAT used by the patients to indicate detailed information on nausea experienced after receiving intravenous chemotherapy. MAT, Multinational Association of Supportive Care in Cancer antiemesis tool.

characteristics placing a patient more at risk of chemotherapy-induced nausea for each subject before initiation of treatment.

\section{Methods}

This prospective, observational study included 100 patients over a seven-month period, in 2017, receiving intravenous chemotherapy at a private oncology centre in South Africa. Of these 100 subjects, 95 diaries were evaluable and used in the study.

\section{Ethical considerations}

This study was conducted in accordance with the Declaration of Helsinki (as revised in 2013). All patients enrolled completed the informed consent form. The study as well as the informed consent document were approved by The Health Research Ethics Committee of NorthWest University, South Africa (NWU-00360-16-S1) before commencing with the study.

\section{Inclusion criteria}

Patients receiving intravenous chemotherapy (chemonaïve patients, as well as patients who have received prior chemotherapy, could take part).

This broad inclusion of patients gave a review of real-life experiences of patients.

\section{Exclusion criteria}

Concomitant radiation therapy and/or surgery within two weeks of chemotherapy treatment.

The study used visual analogue scales (VAS) and patientreported outcome measures (PROMs) to get data to resemble patients' experience as accurately as possible, and to ensure that data was comparable between patients (20).

The tool used for collecting data were based on the Multinational Association of Supportive Care in Cancer (MASCC) antiemesis tool (MAT); it is a validated and standardised tool that was easy to understand and relatively quick to complete (23). The MAT is relied upon for its low patient burden and patient-friendly properties, and measures both acute and delayed nausea and vomiting (24). The directions to complete the diary was explained to the patients before commencement of treatment (Figure 1).

This study focused on the incidence and patterns of nausea in particular. The exact same format for MAT was used, but data were collected on an extended 24-hour table, where the patients could indicate the incidence, duration and intensity of nausea with one pencil marking. 
By collecting the data in this way, it was expected that the results be as close to the real-life experience as possible. The diaries were completed by the patients themselves, aided by the definition of nausea as 'the feeling that you might vomit' and vomiting as 'the expulsion of stomach contents'. Patients documented information in the diaries on the incidence, duration and severity of nausea during the acute phase (0-24 hours), the delayed phase (25-120 hours), day 7 and day 10 after infusion of chemotherapy.

Anticipatory nausea before subsequent cycles, as well as the incidence of vomiting and rescue medication were documented in the diaries. The adapted MAT diaries used a VAS scale that represented 24 hours of the day for the seven days investigated, to measure nausea with ' 0 ' being no nausea experienced at all, to ' 10 ' being nausea at its worst. On this scale, the patient marked exactly when nausea was experienced and its intensity (between $0-10$ ).

Patients were requested to complete diaries for the first three consecutive cycles of their treatment. Ninetyfive patients completed the diaries for cycle one, 87 (92\%) patients completed cycle two and $79(83 \%)$ patients completed diaries for three consecutive cycles. Reasons for some subjects not completing all three first consecutive cycles were disease progression, treatment stopped/changed, patients passed away, non-compliance or withdrawal of consent.

The patients captured their real-life experiences in the diary without any influence from the healthcare providers or clinic. Patients were issued with GCCP, and rescue medication was issued as per published CINV guidelines (22).

Patient demographics were recorded per patient before commencement of treatment, including known risk factors for CINV (age, gender, history of alcohol use in the past two years, history of previous CINV, history of motion sickness, history of morning sickness and the emetogenicity of the chemotherapy utilised).

Other data recorded were medication used to issue breakthrough nausea and/or vomiting (rescue medication), whether patients were issued with prescriptions for rescue medication to take out (TTO) (compared to patients only receiving prescriptions if they required rescue medication) and whether or not patients received proton-pump inhibitors (PPI) with chemotherapy treatment. These variables were tabulated and summarised using descriptive statistics.

\section{Statistical analysis}

All statistical analyses were done in Statistical Package for the Social Science $\left(\right.$ SPSS $\left.^{\circledR}\right)$. All statistical significance was considered with a two-sided probability of $\mathrm{P}<0.05$. The practical significance of results was computed when the $\mathrm{P}$ value was statistically significant $(\mathrm{P} \leq 0.05)$. Variables were expressed using descriptive statistics such as frequencies (n), percentages (\%), means, standard deviations, 95\% confidence interval or medians and interquartile range.

The independent $t$-test (Mann-Whitney U-test) was used to compare the difference between the means of two independent groups. The analysis of variance (ANOVA) (Kruskal-Wallis test) was used for more than 2 groups. If a difference was indicated, a Tukey multiple comparison test was performed to determine which groups differ statistically significantly from one another. Cohen's d value was used to determine the practical significance of the results (with $\mathrm{d} \geq 0.8$ defined as a large effect with practical significance).

Pearson's Chi-square test was used to determine whether an association existed between proportions of two or more categorical variables. The Cramer's V statistic was used to test the practical significance of this association (with Cramer's $V \geq 0.5$ defined as practically significant) (IBM Corp, 2013).

\section{Results}

\section{The incidence of nausea compared to the incidence of vomiting}

One hundred subjects were enrolled over a seven-month period, of which 95 subjects' diaries were evaluable for cycle one (three patients passed away, one patient was noncompliant and one patient was not eligible after screening). The population consisted of 68 females $(71.6 \%)$ and 27 males (28.4\%) between the ages of 24 and 85 years $(\mu=57)$. The ethnicity of the population was made up of seven Indian, 72 Caucasian and 16 African patients.

Patients received a variety of chemotherapy treatments including different emetogenicity levels, consisting of 25 LEC patients (26.3\%), 24 MEC (25.3\%) patients and 46 HEC $(48.4 \%)$ patients. Patients with a variety of cancers were treated, in different stages of disease and performance status scores-the study aimed to create a close as possible representation of real-life data as summarized in Table 1 (25). The only exclusion criteria were concomitant radiation therapy and/or surgery within two weeks of chemotherapy treatment.

The incidence of nausea compared to the incidence of vomiting is captured in Table 2. In this study, $57.9 \%$ of 
Table 1 Characteristics of patients receiving intravenous chemotherapy

\begin{tabular}{|c|c|}
\hline Characteristics & Number $(\mathrm{n}=95)$ \\
\hline \multicolumn{2}{|l|}{ Cancer type } \\
\hline Breast cancer & $47(50 \%)$ \\
\hline Non-Hodgkin's lymphoma & $7(7 \%)$ \\
\hline Prostate cancer & $5(5 \%)$ \\
\hline Lung cancer & $6(6 \%)$ \\
\hline Colon cancer & $11(12 \%)$ \\
\hline Other & $19(20 \%)$ \\
\hline \multicolumn{2}{|l|}{ Stage $(n=95)$} \\
\hline 1 & $4(5 \%)$ \\
\hline II & $26(27 \%)$ \\
\hline III & $23(24 \%)$ \\
\hline IV & $42(44 \%)$ \\
\hline \multicolumn{2}{|l|}{ Performance status $(n=95)$} \\
\hline $\begin{array}{l}0 \text {-fully active, able to carry out all } \\
\text { pre-disease performances without restriction }\end{array}$ & $59(62 \%)$ \\
\hline $\begin{array}{l}\text { 1-restricted in physically strenuous activity } \\
\text { but ambulatory and able to carry out work of a } \\
\text { light or sedentary nature, e.g., light housework, } \\
\text { office work) }\end{array}$ & $29(31 \%)$ \\
\hline $\begin{array}{l}2-\text { ambulatory and capable of all selfcare but } \\
\text { unable to carry out any work activities. Up and } \\
\text { about more than } 50 \% \text { of waking hours }\end{array}$ & $7(7 \%)$ \\
\hline
\end{tabular}

Table 2 The incidence of nausea compared to the incidence of vomiting (overall phase) during cycle 1, 2 and 3

\begin{tabular}{lccc}
\hline & No vomiting & Vomiting & Total \\
\hline Cycle 1 & & & \\
No nausea & $38(95.0 \%)$ & $2(5.0 \%)$ & $40(100 \%)$ \\
Nausea & $34(61.8 \%)$ & $21(38.2 \%)$ & $55(100 \%)$ \\
Total & $72(75.8 \%)$ & $23(24.2 \%)$ & $95(100 \%)$ \\
Cycle 2 & & & \\
No nausea & $43(100.0 \%)$ & $0(0.0 \%)$ & $43(100 \%)$ \\
Nausea & $32(72.7 \%)$ & $12(27.3 \%)$ & $44(100 \%)$ \\
Total & $75(86.2 \%)$ & $12(13.8 \%)$ & $87(100 \%)$ \\
Cycle 3 & & & \\
No nausea & $43(100.0 \%)$ & $0(0.0 \%)$ & $43(100 \%)$ \\
Nausea & $22(61.1 \%)$ & $14(38.2 \%)$ & $36(100 \%)$ \\
Total & $65(82.3 \%)$ & $14(17.7 \%)$ & $79(100 \%)$ \\
\hline
\end{tabular}

patients experienced nausea, of which $61.8 \%$ of those were in the absence of vomiting-only $24.2 \%$ of the patients experienced episodes of vomiting in the overall phase during cycle one. A statistically significant association was found between the incidence of nausea and the incidence of vomiting (in the overall phase) in all three cycles, with $\mathrm{P}<0.05$ (Figure 2).

A practical medium effect was found for both cycle one and two (with phi $\sim 0.382$ and phi $~ 0.395$ ) and a practical large effect for cycle 3 (phi $\sim 0.507$ ).

The incidence of nausea experienced during cycle one was $35.8 \%$ in the acute phase and $56.8 \%$ in the delayed phase, $27.6 \%$ for the acute phase during cycle 2 with $47.7 \%$ in the delayed phase. Cycle three reflected this data with acute nausea recorded as $24.1 \%$ and delayed nausea as $44.3 \%$. Day 7 and day 10 were recorded as a 'late-delayed phase'. Nausea experienced during this time was less than nausea experienced during the overall phase, but still significant in $28.4 \%, 21.8 \%$ and $25.3 \%$ of the patients during the three consecutive cycles.

The mean time to the first incident of nausea was in the delayed phase for all cycles ( $\mu=29.3$ hours). The intensity of nausea (mean) experienced on the VAS was 5.9 out of 10 for cycle one, 6.0 out of 10 for cycle two and 5.9 out of 10 for cycle three. Patients experiencing intermittent nausea had a mean duration of $4.1,3.3$ and 3.8 hours per episode during cycle one, two and three respectively, with $25.8 \%$ of all patients experiencing nausea-experiencing nausea continuously without relief during any cycle.

Vomiting seemed less of a problem during all three cycles, with only $12.6 \%$ patients reporting vomiting incidents during the acute phase for cycle one, $4.6 \%$ during cycle 2 and $3.8 \%$ during cycle 3 . The incidents of vomiting for the delayed phase were $19.5 \%, 8.1 \%$ and $13.8 \%$ for cycle one, two and three consecutively and during the late delayed phase only $5.1 \%$ (cycle one and two); and 3.8\% (cycle three) of patients vomited. The delayed phase had the highest intensity of incidents reported for both nausea and vomiting.

\section{Patient characteristics}

Patient characteristics known to impact the incidence of nausea were recorded with the enrollment of every patient. Statistically significant impact was found with the history of morning sickness $(\mathrm{P}=0.000)$, the history of motion sickness $(\mathrm{P}=0.002)$, age $<60$ years $(\mathrm{P}=0.016)$ and gender of patients $(\mathrm{P}=0.029)$. The practical effect size of this impact was large 


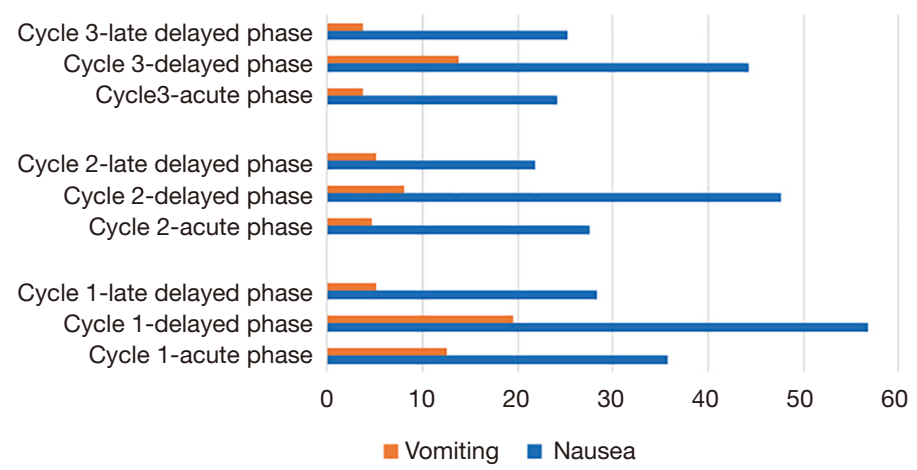

Figure 2 The association between the incidence of nausea and the incidence of vomiting for cycle 1, 2 and 3 .

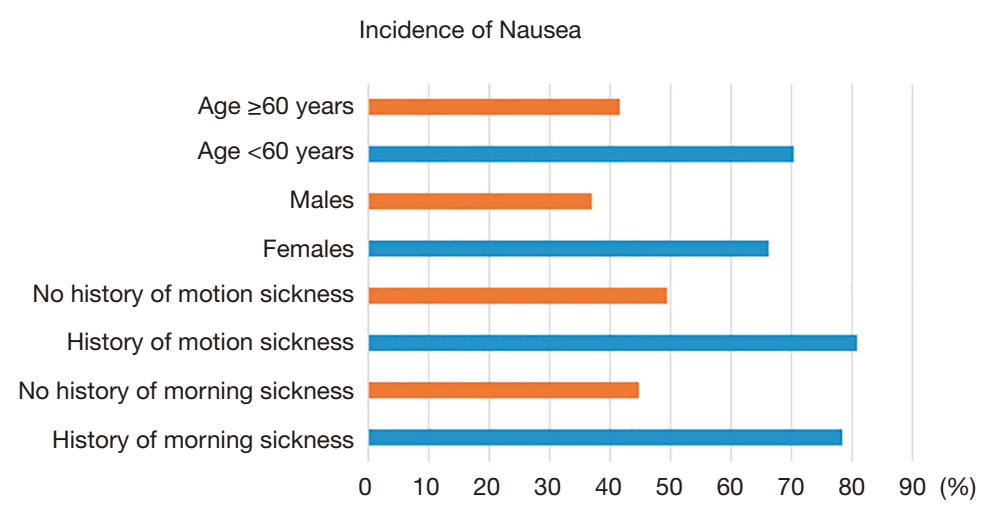

Figure 3 Patient risk-factors with a statistically significant impact on the incidence of nausea.

for patients with a history of motion sickness and a history of morning sickness, and medium for female gender and age $<60$ years (see Figure 3).

Patients with a history of morning sickness had a $78.4 \%$ incidence of nausea with chemotherapy $(\mathrm{P}=0.001)$ and patients with a history of motion sickness showed an incidence of nausea of $80.8 \%(\mathrm{P}=0.006)$. Female patients showed a $66.2 \%$ incidence of nausea compared to only $37.0 \%$ incidence recorded in male patients $(\mathrm{P}=0.009)$. Younger patients ( $<59$ years) showed $70.4 \%$ nausea compared to patients $\geq 60$ years with only $41.5 \%$ incidence of nausea $(\mathrm{P}=0.005)$.

The previous experience of CINV and a history of high alcohol use did not show a statistically significant impact on the incidence of nausea in this study. Patients experienced nausea independently of the emetogenicity of the chemotherapy administered, with LEC patients showing $40.0 \%$ nausea, MEC patients with $62.5 \%$ nausea and $67.4 \%$ of all HEC patients experiencing nausea.

\section{Conclusions}

The broad inclusion criteria of the study created a population with a variety of demographics, disease and treatment. The aim was to create a population as close to the real-life situation of cancer patients as possible. Patients completing their own diaries-indicating their experience of nausea and vomiting-recorded data that were valid and reliable, without misinterpretation from third parties. Data collected with the expanded MAT gave a wealth of information on the patients' experience of nausea and vomiting. Data collected for nausea specifically was valuable-indicating a large part of the population experiencing significant intensities of nausea for hours at a time, with some patients having continuous nausea for the duration for their treatment (despite patients receiving GCCP and rescue medication).

At the time of conducting this study-to the best of the researcher's knowledge-there were no published data focusing on nausea as primary endpoint to compare this 
data to. A statistically significant association between nausea and vomiting was found in all three cycles, as well as an association between the emetogenicity of the treatment and nausea experienced. Despite this association, between $60-70 \%$ of all patients experiencing nausea (during all three cycles) did not report any vomiting incidents.

Patient risk factors impacting nausea reflected in published data $(3,22,26)$, showing an association between the experience of nausea and female gender, age $<60$ years, the history of motion sickness and the history of morning sickness. This information can be used to identify high risk patients, approaching their treatment with strict following of GCCP, clear communication with the patient regarding the management of nausea and their experience of nausea. More studies are needed with nausea as primary endpoint, as well as a standardised tool to measure nausea in a valid and reliable way.

\section{Acknowledgments}

We would like to thank Ms M. Cockeran and Dr. E. Fourie of the North-West University, South Africa for their assistance in the verification of research design, guidance in the interpretation of the results and statistical analysis of the data. Funding: None.

\section{Footnote}

Data Sharing Statement: Available at http://dx.doi. org/10.21037/apm-19-453

Conflicts of Interest: All authors have completed the ICMJE uniform disclosure form (available at http://dx.doi. org/10.21037/apm-19-453). The authors have no conflicts of interest to declare.

Ethical Statement: The authors are accountable for all aspects of the work in ensuring that questions related to the accuracy or integrity of any part of the work are appropriately investigated and resolved. This study was conducted in accordance with the Declaration of Helsinki (as revised in 2013). All patients enrolled completed the informed consent form. The study as well as the informed consent document were approved by The Health Research Ethics Committee of North-West University, South Africa (NWU-00360-16-S1) before commencing with the study.

Open Access Statement: This is an Open Access article distributed in accordance with the Creative Commons Attribution-NonCommercial-NoDerivs 4.0 International License (CC BY-NC-ND 4.0), which permits the noncommercial replication and distribution of the article with the strict proviso that no changes or edits are made and the original work is properly cited (including links to both the formal publication through the relevant DOI and the license). See: https://creativecommons.org/licenses/by-nc-nd/4.0/.

\section{References}

1. Feyer P, Jordan K. Update and new trends in antiemetic therapy: the continuing need for novel therapies. Ann Oncol 2011;22:30-8.

2. Andrews PLR, Sanger GJ. Nausea and the quest for the perfect anti-emetic. Eur J Pharmacol 2014;722:108-21.

3. Gilmore JW, Peacock NW, Gu A, et al. Antiemetic guideline consistency and incidence of chemotherapyinduced nausea and vomiting in US community oncology practice: INSPIRE study. J Oncol Pract 2014;10:68-74.

4. Pirri C, Bayliss E, Trotter J, et al. Nausea still the poor relation in antiemetic therapy? The impact on cancer patients' quality of life and psychological adjustment of nausea, vomiting and appetite loss, individually and concurrently as part of a symptom cluster. Support Care Cancer 2013;21:735-48.

5. Sommariva S, Pongiglione B, Tarricone R. Impact of chemotherapy-induced nausea and vomiting on healthrelated quality of life and resource utilisation: a systematic review. Crit Rev Oncol Hematol 2016;99:13-36.

6. Jordan K, Gralla R, Jahn F, et al. International antiemetic guidelines on chemotherapy-induced nausea and vomiting (CINV): content and implementation in daily routine practice. Eur J Pharmacol 2014;722:197-202.

7. Jordan K, Jahn F, Aapro M. Recent developments in the prevention of chemotherapy-induced nausea and vomiting (CINV): a comprehensive review. Ann Oncol 2015;26:1081-90.

8. Navari RM, Rapoport B, Powers D, et al. Rolapitant for the prevention of nausea in patients receiving highly of moderately emetogenic chemotherapy. Cancer Med 2018;7:2943-50.

9. Moradian S, Howell D. Prevention and management of chemotherapy-induced nausea and vomiting. Int J Palliat Nurs 2015;21:216-24.

10. Cohen L, de Moor CA, Eisenberg P, et al. Chemotherapyinduced nausea and vomiting - incidence and impact on patient quality of life at community oncology settings. 
Support Care Cancer 2007;15:497-503.

11. dos Santos LV, Souza FH, Brunetto AT, et al. Neurokin-1 receptor antagonists for chemotherapy-induced nausea and vomiting: a systematic review. J Natl Cancer Inst 2012;104:1280-92.

12. Roila F, Molassiotis A, Herrstedt J, et al. MASCC and ESMO guideline update for the prevention of chemotherapy- and radiotherapy-induced nausea and vomiting and of nausea and vomiting in advanced cancer patients. Ann Oncol 2016;27:v119-33.

13. Navari RM. Olanzapine for the prevention and treatment of chronic nausea and chemotherapy-induced nausea and vomiting. Eur J Pharmacol 2014;722:180-6.

14. Abe M, Kasamatsu Y, Kado N, et al. Efficacy of olanzapine combined therapy for patients receiving highly emetogenic chemotherapy resistant to standard antiemetic therapy. Biomed Res Int 2015;2015:956785.

15. Aapro M, Molassiotis A, Dicato M, et al. The effect of guideline-consistent antiemetic therapy on chemotherapyinduced nausea and vomiting (CINV): The Pan European Emesis Registry (PEER). Ann Oncol 2012;23:1986-92.

16. Bashashati M, McCallum RW. Neurochemical mechanisms and pharmacologic strategies in managing nausea and vomiting related to cyclic vomiting syndrome and other gastrointestinal disorders. Eur J Pharmacol 2014;722:79-94.

17. Andrews PLR, Horn CC. Signals for nausea and emesis: Implications for models of upper gastrointestinal diseases. Auton Neurosci 2006;125:100-15.

18. Babic T, Browing KN. The role of vagal neurocircuits in the regulation of nausea and vomiting. Eur J Pharmacol

Cite this article as: Smit T, Kotze I, du Plessis J. The incidence of nausea in the absence of vomiting in patients receiving intravenous chemotherapy. Ann Palliat Med 2021;10(3):2679-2686. doi: 10.21037/apm-19-453
2014;722:38-47.

19. Chin CL, Fox GB, Hradil VP, et al. Pharmacological MRI in awake rats reveals neural activity in area postrema and nucleus tractus solitarius: Relevance as a potential biomarker for detecting drug-induced emesis. Neuroimage 2006;33:1152-60.

20. Pleuvry BJ. Physiology and pharmacology of nausea and vomiting. Anaesth Intensive Care 2015;16:462-6.

21. Ballatori E, Roila F. Impact of nausea and vomiting on quality of life in cancer patients duringchemotherapy. Health Qual Life Outcomes 2003;1:46.

22. Gozzo Tde O, de Souza SG, Moysés AM, et al. Incidence and management of chemotherapy-induced nausea and vomiting in women with breast cancer. Rev Gaucha Enferm 2014;35:117-23.

23. Brink H, van der Walt C, van Rensburg G. Fundamentals of research methodology for healthcare professionals. Juta, Cape Town, 2012.

24. Roila F, Herrstedt J, Aapro M, et al. Guideline update for MASCC and ESMO in the prevention of chemotherapyand radiotherapy-induced nausea and vomiting: results of the Perugia consensus conference. Ann Oncol 2010;21:v232-43.

25. Oken MM, Creech RH, Tormey DC, et al. Toxicity and response criteria of the Eastern Cooperative oncology Group. Am J Clin Oncol 1982;5:649-55.

26. Waqar SN, Mann J, Baggstrom MQ, et al. Delayed nausea and vomiting from carboplatin doublet chemotherapy. Acta Oncol 2016;55:700-4. 